\title{
Comparative Study Of Children's Current Health Conditions And Health Education In New Zealand And Japan
}

Kanae Watanabe, Aoyama Gakuin Women's Junior College, Japan

Annette Dickinson, Auckland University of Technology, New Zealand

\begin{abstract}
In New Zealand (NZ) and Japan, despite comprehensive national health and physical education (HPE) curriculums in schools, there continues to be significant health issues for children. A qualitative interpretative descriptive research method was used to compare how primary school teachers taught HPE in both countries. In NZ, there is some freedom in relation to the school interprets and delivers HPE. However, there is disparity in how the curriculum is delivered between schools in low and high socio-economic areas. In Japan, the government directs the delivery of HPE through government-designated textbooks. Therefore, while there is no disparity between schools, teachers cannot customize health education according to their students' needs. The flexibility of HPE in NZ is both an advantage in that it enables a creative and innovative teaching approach customized to the community in which the school is situated and a disadvantage in that health education is decided not according to the needs of the children but according to the financial resources and teachers' enthusiasm. In Japan, while children receive health education that may be useful for the future, the HPE curriculum does not address current child health issues. Moreover, it is difficult to teach all the content within the governmentdesigned HPE textbook within official designed time for health education. This study's results suggest that both countries need to review the delivery and resourcing of their HPE to ensure that children receive education that addresses their current and future health needs and those of their families.
\end{abstract}

Keywords: Children; Health Education; New Zealand; Japan

\section{INTRODUCTION}

1 children in NZ The National Heart Foundation of New Zealand, 2007). In Japan, insufficient sleep, polarization of child fitness due to quantity of exercise, inadequate food intake, mental health issues, and experiences with abuse are reported as the main health issues experienced by children (Japanese Society of School Health, 2014; Science Council of Japan, 2010). In both NZ (Ministry of Education New Zealand (a), 2007; (b), 2007) and Japan (Ministry of Education, Culture, Sports, Science and Technology Japan, 2008), national health and physical education (HPE) curriculums guide the teaching HPE in schools. Despite teachers' adherence to HPE as one way of improving the health of children significant child health issues persist. This study aimed to examine child health and health education issues in NZ and Japan and to offer recommendations for the improvement and development of health education for children in both countries by answering the following research questions:

n New Zealand (NZ), obesity/overweightness and asthma are known child health issues, and there are marked ethnic and socioeconomic inequities (Ministry of Health, New Zealand, 2012). For example, rheumatic fever is still a serious child health issue among particular ethnic groups; Maori and Pacific 
1. What is the perception among teachers in both countries regarding the health issues that affect the children they teach?

2. What health education is delivered to schoolchildren, and what are the outcomes of that education?

3. What is the influence of the HPE curriculum and the education system on the health education provided in schools?

\section{METHODS}

A qualitative interpretive descriptive design was used. Semi-structured interviews with teachers from five compulsory schools in both NZ and Japan (Table 1). One author (KW) interviewed the NZ schoolteachers in English and the Japanese teachers in Japanese. All interviews were audiotaped and transcribed, Data analysis was undertaken by both authors data using Braun and Clarke's (2006) ${ }^{1)}$ framework for thematic analysis.

Table 1: Participants (Interviewed)

\begin{tabular}{lll}
\hline & \multicolumn{1}{c}{ New Zealand } & \multicolumn{1}{c}{ Japan } \\
\hline School & 4 public primary schools/1 public intermediate school & 5 public primary schools \\
\hline Area & Auckland & Tokyo, Kanagawa (next to Tokyo) \\
\hline Decile rating & $\begin{array}{l}\text { Decile 1,4,7, and 10 primary (each) } \\
\text { Decile 10 intermediate }\end{array}$ & - (No comparable rating in Japan) \\
\hline Teacher & 4 females, 1 male & 2 females, 3 males \\
\hline
\end{tabular}

The semi-structured interviews were guided by the following questions:

1. What are the health issues faced by the children in your class?

2. What do you think are the main causes of these health issues?

3. What do you do in your classroom in relation to health education?

4. Is this education guided by a health curriculum?

5. Do you think the health curriculum is adequate for improving the health of the children in your classroom?

6. What support do you need in order to carry out your health education?

7. What do you think are the outcomes for the health education received by the children in your classroom?

8. How do you think the health curriculum and/or education could be developed?

The researched was approved by the Auckland University of Technology Ethics Committee (AUTEC 13./166) for the New Zealand phase of the study and in Japan complied with the guidelines of the Japan Society of Physical Education Health and Sports Science, Ethics of Researchers, 2011.

\section{FINDINGS}

\section{Children's Health Issues As Identified by The Teachers}

New Zealand

In NZ, one of the most frequent child health issues identified by the teachers was obesity/overweightness. Low decile rating (decile) schoolteachers said that there were few obese children(less than $10 \%$ of all children) in their schools. However, from the researchers personal observations of their classrooms and schools, there were many obese children (about $20 \%$ of all children). High decile schoolteachers told us that there were very few, or no, obese children in their schools. However, from the researchers personal observation, in some classes there was no obese children, but in other classes, $5-10 \%$ of the children were obese.

Another frequently identified child health issue was asthma. Both in the low and high decile schools, there were some children with asthma. The low decile schoolteachers' perceptions of the possible causes of this asthma were poor housing, damp and overcrowded homes that lacked adequate insulation. Low decile schoolteachers reported that many of their children lived in poor housing conditions. However, high decile schoolteachers did not perceive that the living conditions of their students affected asthma incidence. Therefore, the causes of asthma 
among the high decile schoolchildren could not be explained by housing conditions. They said that allergies might be one possibility but they were not sure.

In NZ, Ministry of Health and public health nurses clearly identified rheumatic fever as an issue in their reports and in information circulated to the schools (The National Heart Foundation of New Zealand, 2007). However, teachers did not identify, or even know about rheumatic fever, even for the students who belonged to high-risk groups and schools in high-risk communities.

The findings concerning NZ children's health were as follows: while teachers had some understanding of obesity/overweightness and asthma as health issues, their knowledge in regards to the health issues faced by the community in which they taught was poor.

Japan

In Japan, the most frequent child health issues as identified by teachers were insufficient sleep and inadequate food intake. In addition, some teachers commented about the children's poor fitness and about the polarization, they saw between unfit and well-fit children in their classrooms. The common reasons cited for insufficient sleep and low-fitness were console games, smartphones, social network services (SNS), busyness in cramming for school, and/or lessons after school. Teachers noticed that children began playing more inside instead of outside, and console games, smartphones, and SNS were the main activities engaged in during indoor play. These observations are supported by the official reports of children's health and lifestyle trends (Japanese Society of School Health, 2014; Science Council of Japan, 2010). Teachers said that the students' inadequate food intake was the result of fastidiousness about food and Epicureanism.

The findings about teachers' perceptions of children's health in Japan related to lifestyle and habits, which are formed, controlled, and affected by parents' lifestyle choices and by home discipline. Namely, Japanese children's current health conditions and issues might be influenced by their parent-child relationships. The teachers believed that children with families who live a healthy lifestyle did not need health education in schools however children growing up in the opposite type of family environment need to be educated about healthy lifestyles..

\section{Health Education Delivery by School Teachers}

New Zealand (Table 2)

All of the schoolchildren participants learned about healthy food and exercise. This was directly related to obesity/overweightness, which the teachers identified as the main child health issue in their classrooms.

Table 2: Location, equipment, and support of low and high decile schools' education

\begin{tabular}{|c|c|c|c|c|}
\hline & Location & Equipment and support & Lesson style & Resources \\
\hline $\begin{array}{l}\text { Low } \\
\text { decile } \\
\text { schools }\end{array}$ & $\begin{array}{l}\text { Only in } \\
\text { schools }\end{array}$ & $\begin{array}{l}\text { Without additional costs } \\
\text { Without parental support } \\
\text { (Even though there was no cost for the } \\
\text { Schools) }\end{array}$ & Lecture only & $\begin{array}{l}\text { - Cost-free educators } \\
\text { - Amateur, volunteer coaches }\end{array}$ \\
\hline \multirow{3}{*}{$\begin{array}{l}\text { High } \\
\text { decile } \\
\text { schools }\end{array}$} & In schools & $\begin{array}{l}\text { with and without additional costs } \\
\text { - Special gear } \\
\text { - Professional coaches }\end{array}$ & \multirow{3}{*}{$\begin{array}{l}\text { Lecture and } \\
\text { activities } \\
\text { (Experiences) }\end{array}$} & \multirow{3}{*}{$\begin{array}{l}\text { Not only cost-free educators but } \\
\text { also professional coaches and } \\
\text { instructors }\end{array}$} \\
\hline & $\begin{array}{l}\text { Events in } \\
\text { and around } \\
\text { schools }\end{array}$ & $\begin{array}{l}\text { With additional costs } \\
\text { With parents' support } \\
\text { (Manpower, donations, provisions) }\end{array}$ & & \\
\hline & $\begin{array}{l}\text { Outdoor } \\
\text { activities }\end{array}$ & $\begin{array}{l}\text { With additional costs } \\
\text { - Special gear } \\
\text { - Professional support } \\
\text { With parents' support }\end{array}$ & & \\
\hline
\end{tabular}


Low decile schools offered HPE only within the school using activities that did not incur additional costs. They needed parental support, but this was not always available. Parents could not come to school during the daytime because of paid work or family caregiving commitments. As for lesson style, low decile schools' lessons were mainly lecture-only. Low decile schools could use only cost-free educators; for example, police education officers, nurses, Life Education Caravan, and so on and relied on amateur volunteers to participate as coaches.

In High decile schools' HPE was performed not only in the schools but also in the community and at outdoor locations. For example, A Health Festival was held at the school in the weekend with parents and neighboring residents being invited to join in health events with the students. While these schools did use cost-free activities and activities they were also able to pay for additional resources and teaching including special sports gear, like hockey sticks and uniforms and invited professional sports coaches

In NZ, there were large disparities between the low and high decile schools in terms of the activities offered to the children,. High decile schoolchildren had many opportunities to apply their knowledge and learned skills through activities, while low decile schools did not. Teachers noted that the outcomes of HPE were not specific to one health issues or topic but holistic, not focused on technique but on attitude, and therefore difficult to measure or to evaluate objectively. The most frequently mentioned advantages were children leaving schools as well-rounded individuals and knowing how to manage themselves and how to relate to other people. The second most frequently mentioned advantage of HPE was that children had good attitudes toward fitness.

Japan

In this country, the national curriculum requirements prescribes what, when, and how each subject could be taught within the schools. All primary school students study the same subjects in the same grade (school year) and in the same way using government (Ministry of Education, Culture, Sports and Technology Japan) -authorized textbooks conforming to the national curriculum. Therefore, no health education disparities were observed. Every child had access to health education that met the standardized acceptable level. Almost all health education lessons were presented in a lecture style, so the children could get important health knowledge; however, sometimes there was an overemphasis on knowledge and a lack of practical training.

Children learned about three health topics: nutrition, exercise, and rest (sleep) during the 3rd and 4th grades (at ages 9-10 years old). They obtained this knowledge through textbook lectures, but they did not have the opportunity to apply their knowledge through activities. It was the perception of the teachers that these health topics were concerned with lifestyle which is often influenced by parental values and therefore it was difficult for the children to realize the importance of the knowledge they obtained in their health classes. Another oversight of the program was issues related to screen time, the time spent with console games, smartphones, and SNS was not addressed by the teachers despite the fact that this was identified as a major health issue amongst Japanese children. This was because this is not written in either the HPE national curriculum or the textbooks. Moreover as one teacher noted, there appears to be some difference in opinion related to whose responsibility it is to teach the necessary discipline concerned with lifestyles and habits, with the teachers believing these topics should be taught at home while parents expect that these issues will be addressed in school. Some teachers said that this tendency had grown increasingly stronger.

Many teachers did not identify what the expected outcomes of HPE during childhood however they did expect that learning health education in school might prove useful in the future life. They believed that although there may be an emphasis on knowledge versus application this could lead to significant health improvements because Japanese adults would be more knowledgeable about health issues. 


\title{
HPE Curriculum And Education System
}

\author{
New Zealand
}

NZ schoolteachers perceived the HPE curriculum as being flexible and open, and to use and able to be individualize for each school. This, was both an advantage and a disadvantage. The advantage was that schools could prioritize the topics covered each year based on school and health priorities. The disadvantage was that each school's health education was decided not by the children's needs but by the available financial resources and teachers' enthusiasm and motivation for teaching health education. Even low-quality educational lessons could meet curriculum objectives with the amount of time teachers spent teaching health education being variable.

Difficulties concerning health education centered on the following curriculum and education system issues: (1) Time for health education was limited; (2) Academic teaching - for example, reading, writing, and mathematics - took priority over health education; and (3) Unlike reading, writing, and mathematics, there was no measured or reported national standard of health education.

\section{Japan}

The strictly prescribed Japanese curriculum and government-authorized textbooks guaranteed consistency in the quality and delivery of health education no matter which school the children attended. Children were able to gain more knowledge of both health and health science than NZ children were. . However, a disadvantage was that the currency and responsiveness of curriculum to child health issues. The national curriculum is only reviewed every ten years and therefore the teachers noted that the topics they must teach might not relate to current child health issues or the needs of the community in which the children lived. For example, teachers anxious about children's spending too much time on console games, smartphones, and SNS because those might be the causes of children's lack of sleep and exercise could not teach on this issue at all because these things are not mentioned in the HPE curriculum.

Like NZ, the teachers noted the priority of academic teaching over health education. Despite Japan having regulated lesson time this was not sufficient to cover the content written in the HPE curriculum and textbooks. Therefore, teachers always feel rushed when covering health education. Moreover, classroom teachers currently take charge of health education, but they need the support of health professionals (e.g., medical doctors, police officers, pharmacists, etc.), experienced citizens (pregnant women, ex-smokers, etc.).

\section{CONCLUSION}

The NZ HPE curriculum and education system provides a good platform for school health education. In particular, children in high decile schools were offered varied and interesting programs to ensure their health, not only through lectures but also through activities. However, there were disparities in relation to the variety and quality of programs and activities offered across all NZ schools with often children living in areas of high health need receiving the lowest quality health education in school.

The Japanese HPE curriculum and education system guaranteed the quality of health education and showed no educational disparities between students. However, teachers were unable to teach about the relevant current health issues affecting their students because those issues were not prescribed within the HPE curriculum.

\section{RECOMMENDATION}

We recommend the following improvements be made to children's health education in NZ and Japan.

\section{In New Zealand}

Professional teacher training regarding HPE needs to be resourced and prioritized especially in low decile schools. The Ministry of Education also needs to consider prioritizing HPE in low decile schools which often are 
situated in communities which have the poorest health outcomes for children. These schools need better access to high-quality free resources and educational specialists. Teachers need to made more aware of child health issues and be encouraged to teach and deliver health education that meets the needs of the community in which they teach. It is may also be necessary to consider national standards for HPE, as is done for reading, writing, and mathematics to ensure that the quality of HPE is monitored across schools.

\section{In Japan}

The Ministry of Education, Culture, Sports and Technology Japan needs to consider reducing the compulsory contents of the HPE curriculum and textbooks provide health education that gives the teachers more latitude and be more responsive to the health needs of their communities. A less prescribed HPE curriculum may allow teachers to be more flexible and creative with their lessons with more opportunities for the application of knowledge outside of the classroom. HPE in Japan might also benefit from the inclusion of teaching support from the wider health professional community and more active involvement and inclusion of parents. Any changes would need to be supported by Professional education of teachers in the delivery of a more flexible curriculum.

\section{ACKNOWLEDGEMENT}

This research was supported by the Japan Society for the Promotion of Science (JSPS) Grants-in-Aid for Scientific Research (C), Grant Number 24600025. Dr. Tineke Water, Dr. Andrew Gibbons and Dr. Leon W. Benade of Auckland University of Technology helped us carry out our interviews and gave us important information about New Zealand's school education system and curriculum.

\section{REFERENCES}

Braun V., Clarke V. (2006). Using thematic analysis in psychology. Qual Res Psychol, 3:2, 77-101.

Japanese Society of School Health (2014). Surveillance of children's health status, 4-12, Tokyo: Katsumi Print Co. Ltd. (In Japanese).

Ministry of Education, Culture, Sports, Science and Technology Japan. (2008). Course of Study for Elementary Schools, Section 9 Physical Education,1-13 (English version).

Ministry of Education New Zealand (a). (2007). Curriculum achievement objectives by learning area, Health and Physical Education, 14-17, Wellington: Learning Media Limited.

Ministry of Education New Zealand (b). (2007). New Zealand Curriculum, Health and Physical Education.22-23, Wellington : Learning Media Limited.

Ministry of Health, New Zealand. (2012). The health of New Zealand children 2011/12: Key findings of the New Zealand Health Survey, 13-29.

Science Council of Japan. (2010). Japanese child health promotion, 4-20.

The National Heart Foundation of New Zealand. (2007). A summary of the New Zealand guidelines for rheumatic fever, 1-4. 